\section{Vitreous and retinal amino acid concentrations in experimental central retinal artery occlusion in the primate}

\author{
Abstract \\ Purpose Vitreous and retinal amino-acid \\ concentrations were evaluated in a primate \\ model of central retinal artery occlusion \\ (CRAO) to study the role of glutamate \\ excitotoxicity in acute retinal ischaemia. \\ Methods Unilateral, acute CRAO was \\ produced by temporary clamping of the \\ central retinal artery for $\mathbf{1 9 0} \mathbf{~ m i n}$ in four elderly \\ rhesus monkeys. Fundus photography, \\ fluorescein angiography, and \\ electroretinogram were performed before and \\ during CRAO, and after unclamping the \\ artery. Vitreous samples were obtained before \\ and after CRAO in both eyes, and analysed for \\ 13 amino-acid concentrations using high- \\ pressure liquid chromatography. The animals \\ were killed $350 \mathrm{~min}$ after retinal reperfusion, \\ and the retinal tissue was submitted for \\ amino-acid analysis. \\ Results In all four eyes, the macula showed \\ the 'cherry red spot'. The CRAO was \\ confirmed by fluorescein angiography and \\ decreased b-wave on electroretinogram. \\ Retinal histology confirmed ischaemic \\ changes in the inner retina. Changes in all 13 \\ vitreous amino-acid concentrations after \\ CRAO (including glutamate) were not \\ significantly different between study and \\ control eyes $(P=0.09$ to 0.82$)$. All retinal \\ amino-acid concentrations (including \\ glutamate) were not significantly different \\ between two eyes $(P=0.07-0.93)$. \\ Conclusions In the primate model of acute \\ inner retinal ischaemia induced by transient \\ CRAO, we were unable to detect significantly \\ elevated concentrations of vitreous and \\ retinal glutamate. Our primate model
}

YH Kwon', DW Rickman', S Baruah1, MB Zimmerman², C-S Kim', HC Boldt', SR Russell ${ }^{1}$ and SS Hayreh' ${ }^{1}$

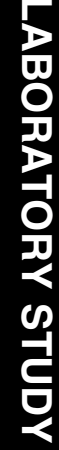

has the advantage of closely modelling the CRAO in humans. Further basic and clinical studies are needed to elucidate the role of glutamate excitotoxicity in retinal ischaemia.

Eye (2005) 19, 455-463. doi:10.1038/sj.eye.6701546

Published online 21 May 2004

Keywords: amino acid; glutamate; central retinal artery occlusion; primate; retinal ischaemia

\section{Introduction}

Central retinal artery occlusion (CRAO) produces ischaemia of the inner retina and often leads to significant loss of vision. Detailed experimental studies in Rhesus monkeys have shown that CRAO for less than $100 \mathrm{~min}$ produced little or no apparent retinal damage. However, CRAO of 105-240 min produced a variable degree of damage, while almost total irreversible damage was observed with CRAO for longer than $240 \mathrm{~min} .{ }^{1,2}$ Various treatments aiming at restoring retinal blood circulation in CRAO have been described. ${ }^{3}$ Despite anecdotal reports of visual improvement following treatments, ${ }^{4}$ it is presently unclear whether any of the treatments significantly alters the natural outcome of the disease. $^{5}$

Glutamate is an amino acid essential for cellular metabolism. It is also an excitatory neurotransmitter found in the central nervous system including the retina. ${ }^{6}$ Interestingly, excess levels of glutamate can be neurotoxic (referred to as 'excitotoxicity'). ${ }^{7}$ The glutamate excitotoxicity has been found to be a mechanism of cell death in acute brain ischaemia. ${ }^{7}$

Furthermore, it has been proposed as a
${ }^{1}$ Department of Ophthalmology \& Visua Sciences University of lowa lowa City, IW, USA

${ }^{2}$ Department of Biostatistics University of lowa lowa City, IW, USA

Correspondence: YH Kwon Department of Ophthalmology and Visual Sciences

University of lowa Hospitals and Clinics 200 Hawkins Dr. lowa City, IW 52242, USA Tel: + 11319 356-3933 Fax: + 113193537699

E-mail: young-kwon@ uiowa.edu

Received: 23 October 2003 Accepted: 1 March 2004 Published online: 21 May 2004

Presented in part at the Association for Research in Vision and Ophthalmology meeting, Fort Lauderdale, FL, April 2001

Commercial relationships: None for all authors 
mechanism of neuronal death in acute retinal ischaemia. ${ }^{8}$ Indeed, experimental studies show retinal ischaemic damage can be mitigated by glutamate receptor antagonists. ${ }^{9,10}$ These studies collectively suggest that retinal ischaemic damage is, at least partially, mediated by glutamate excitotoxicity. If so, glutamate antagonists may provide a novel treatment strategy for acute CRAO, as suggested previously. ${ }^{8}$

The current study was undertaken to provide a direct evidence for glutamate excitotoxicity in acute retinal ischaemia as a part of an effort to develop a neuroprotective treatment strategy. We studied the role of glutamate excitotoxicity, by measuring vitreous and retinal amino-acid levels in an experimental model of CRAO. We utilized a primate model of acute CRAO, which closely mimics the human condition. ${ }^{11}$

\section{Materials and methods}

The study was conducted in four elderly (average age of 20 years) Rhesus monkeys. Acute CRAO was induced in one eye, and the fellow eye served as control. The study design complied with the National Institute of Health's as well as the University of Iowa's Institutional Guidelines for the Care and Use of Laboratory Animals, and ARVO Statement for the Use of Animals in Ophthalmic and Vision Research.

\section{Induction of CRAO}

Detailed experimental protocol for inducing transient CRAO in monkeys has previously been described by one of us. ${ }^{11}$ Briefly, a lateral orbitotomy was performed under general anaesthesia induced by intravenous pentobarbitone sodium. The lateral rectus muscle was detached from its insertion to the globe. The optic nerve was isolated, and the central retinal artery (CRA) was identified at its entrance to the optic nerve using an operating microscope. A microclamp was applied near the point of its entry into the optic nerve and the wound was temporally closed. After the desired period of CRAO (approximately $3 \mathrm{~h}$ ), the microclamp was removed and the blood was allowed to reperfuse the retina for approximately $6 \mathrm{~h}$ before the animal was killed. Both eyes were enucleated for histologic evaluation. The animal remained under intravenous general anaesthesia during the entire experimental period until the killing.

\section{Confirmation of CRAO}

Prior to the day of experiment, all monkeys underwent a baseline ophthalmic evaluation including ophthalmoscopy, fundus photography, fundus fluorescein angiogram, and electroretinogram (ERG) under general anaesthesia, to ensure both eyes were normal. We obtained full-field, scotopic (with $30 \mathrm{~min}$ of dark adaptation) and photopic flash ERG. Monkeys with any ocular abnormality including cataract were excluded from the study. On the day of experiment, a second set of fundus photographs, fluorescein angiograms, and ERG was obtained after the CRA was clamped, as described above. Finally, a third set was obtained after the CRA was unclamped. These evaluations were performed to confirm the CRAO and subsequent reperfusion of the retina.

\section{Vitreous sample collection}

After the completion of the lateral orbitotomy but before the induction of CRAO, a standard two-port, automated pars plana vitrectomy was performed to obtain posterior vitreous specimen from both eyes. Separation of the posterior hyaloid membrane was avoided because doing so may disrupt the inner retinal surface and vessels and potentially cause retinal or optic nerve damage as well as vitreous haemorrhage. $^{12}$ It was necessary to obtain enough volume of vitreous (approximately 200-250 $\mu \mathrm{l}$ ) to reliably transfer the sample into vials for subsequent processing (see below). No irrigating solution was used during vitrectomy, and care was taken to avoid vitreous haemorrhage from the pars plana incision. After the vitreous sample was obtained, equal amount of Balanced Salt Solution (Alcon Laboratories, Inc., Fort Worth, TX, USA) was infused into the anterior chamber through a separate paracentesis wound to reform the eye. The sclerostomy wound was temporarily closed.

The same vitrectomy procedure was repeated in both eyes after approximately $6 \mathrm{~h}$ of reperfusion of the retina following CRAO, just before the animal was killed. In one animal, multiple vitreous biopsies were performed both during and after CRAO. The vitreous samples were placed on ice, taken to the laboratory, and centrifuged for 3 min to separate any (potential) cellular components from the liquid vitreous. Only the liquid supernatant was saved and stored at $-70^{\circ} \mathrm{C}$ until the time of amino-acid analysis.

\section{Tissue preparation and histology}

At the end of the experiment, both eyes were enucleated. The anterior segment (anterior to pars plana) was first removed and the posterior segment was flattened into four quadrants centered at the fovea, with relaxing cuts. Retinal tissue from three of the four quadrants (approximately $5 \times 5 \mathrm{~mm}$ each) was teased away from the underlying tissue using a fine brush and placed into a tissue fixative. Retinal histology was performed using methylene blue stain. The retinal tissue from the 
remaining quadrant was frozen at $-70^{\circ} \mathrm{C}$ for subsequent homogenization and amino-acid analysis.

\section{Amino-acid analysis}

Amino-acid analysis was performed in the Department of Psychiatry at the University of Iowa. The analysis was performed in batches using only the sample number, without the knowledge of the study protocol. The retinal tissue was thawed and homogenized in an isolation medium buffer $(0.32 \mathrm{M}$ sucrose, $1.0 \mathrm{mM}$ EDTA, $10 \mathrm{mM}$ Tris-HCl; pH 7.4) using a motorized Teflon pestle. The homogenate was aliquotted for amino-acid analysis. In all, 13 amino acids, including asparagine (Asn), gammaaminobutyrate (GABA), glutamine (Gln), glutamate (Glu), glycine (Gly), histidine (His), isoleucine (Ileu), leucine (Leu), methionine (Met), phenylalanine (Phe), serine (Ser), threonine (Thr), and tyrosine (Tyr) were analysed using high-pressure liquid chromatography (HPLC) as described previously by one of us. ${ }^{13}$ Briefly, concentrations of amino acids were determined by first adding $200 \mathrm{nmol}$ of allothreonine (Sigma, St Louis, MO, USA) as internal standard, and then deproteinizing with $0.20 \mathrm{ml}$ of $50 \mathrm{mM}$ picric acid. After centrifugation at $16,000 \mathrm{~g}$ for $3 \mathrm{~min}$, the supernatant was filtered through a $0.22 \mu \mathrm{m}$ filter (Millipore, Bedford, MA, USA). Amino-acid analysis was performed following precolumn derivatization with ophthaldehyde (OPA; Fluka, Ronkoncoma, NY, USA). In all, $20 \mu$ l samples were mixed with $60 \mu \mathrm{l}$ of $2.0 \mathrm{mg} / \mathrm{ml}$ solution of OPA containing $0.01 \%$ solution of 2-mercaptopropionic acid (Fluka). Samples were derivatized for $2.0 \mathrm{~min}$ prior to being injected into a reverse-phase $\mathrm{C}-18$ Altima column $(5 \mathrm{~mm}$, $15 \mathrm{~cm} \times 4.6 \mathrm{~cm}$; Alltech, Waukegan, IL, USA). A Gilson model 231 autosampler (Middleton, WI, USA) was routinely used for derivatization and injection. The column was maintained at $40^{\circ} \mathrm{C}$. The variable mobile phases were $50 \mathrm{mM}$ sodium acetate ( $\mathrm{pH}$ 5.65) with $4 \%$ acetonitrile and methanol. The HPLC consisted of a SCL-6A system controller, two LC-6A pumps, and a RF-535 fluorescence detector (Shimadzu, Columbia, MD, USA). The detector was set at an excitation wavelength of $345 \mathrm{~nm}$ and an emission wavelength of $445 \mathrm{~nm}$. Data were collected and analysed using a Chromatopac C-R4A integrator (Shimadzu).

\section{Reliability of amino-acid analysis}

Vitreous fluids were analysed by HPLC in several batches over a period of 12 months. Multiple calibration standards were included in each batch to be analysed. As a measure of variation, the coefficient of variation (CV) was calculated based on $50 \mu \mathrm{M}$ calibration standards repeated 12 different times. The CV was lowest for Phe
(2.6\%), and highest for Gln (28.7\%). The CV for Glu was $6.6 \%$. The CV for the other amino acids ranged from $5.7 \%$ (His) to $10.7 \%$ (Gly and Ser).

\section{Statistical analysis}

The vitreous amino-acid levels were compared between the control and study (CRAO) eye before and after occlusion using the repeated measures ANOVA. The two factors in this analysis were condition of eye (control or CRAO) and time (pre and postocclusion). This analysis compared the change in amino-acid levels from pre- to post-occlusion between the control and CRAO eye. For the retinal amino-acid levels, the comparison between the control and the CRAO within the same monkey was made using the paired $t$-test. A Bonferroni adjustment for multiple amino-acid comparisons was considered; however, it was not employed because it did not change the outcome of the results (see Results). Prior to the analysis, the natural logarithm transformation was applied to normalize the data distribution. In addition, a power analysis was performed to compute a minimum change in the amino-acid levels for a significant change.

\section{Results}

Experimental CRAO was induced for $190 \pm 8 \mathrm{~min}$ in four eyes of four elderly monkeys. In all the four study eyes, the macula showed the classic 'cherry red spot' consistent with the clinical diagnosis of acute CRAO (Figure 1). Occlusion of the central retinal artery with the microclamp in position was confirmed by fundus fluorescein angiography (Figure 1). Full-field scotopic ERG obtained during CRAO showed significant b-wave reduction and resulted in an electronegative wave (Figure 2). Following the CRAO, the retinal circulation was restored to normal for $350 \pm 30 \mathrm{~min}$ before the animal was killed. Retinal histology in the CRAO eye demonstrated abnormal, swollen inner nuclear, ganglion cell, and nerve fibre layers with sparing of the outer nuclear and photoreceptors layers, indicating ischaemic insult confined to the inner retina supplied by the central retinal artery (Figure 3).

Amino-acid concentrations in the vitreous and retina of the study (CRAO) and control (fellow) eyes are shown in Table 1. There was no significant difference in the baseline vitreous concentrations of amino acids between control and study eyes $(P=0.22$ to $>0.99$, paired $t$-test, Table 1). The changes in the vitreous amino-acid concentrations after CRAO were not significantly different between the two eyes $(P=0.09-0.82$, repeated measures ANOVA, Table 1). In particular, vitreous glutamate levels decreased by 34.5 and $20.5 \%$ in the control and CRAO eye, respectively $(P=0.43$, repeated 

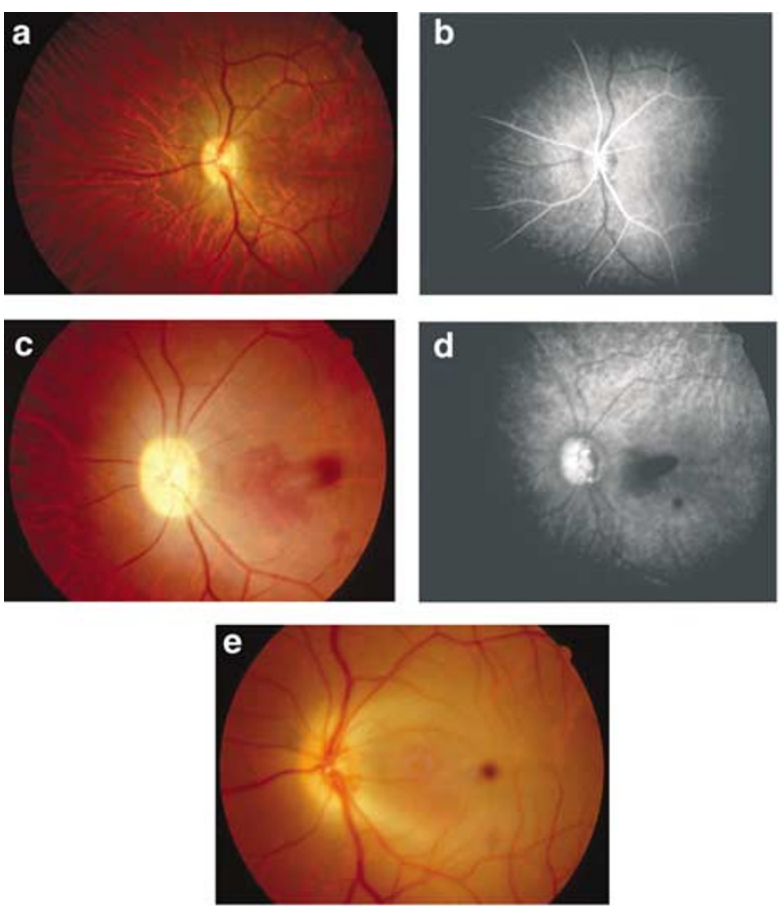

Figure 1 Fundus photographs and fluorescein angiograms of a left eye before, during, and after CRAO. Before CRAO, the fundus is normal (a) with normal retinal arterial perfusion $8.9 \mathrm{~s}$ after peripheral intravenous fluorescein injection (b). During CRAO, the macula begins to opacify with narrowing of the arterioles (c). During CRAO, a fluorescein angiogram shows absence of retinal arterial filling at $21.2 \mathrm{~s}$ after the dye injection (d). After CRAO, the macula shows a 'cherry-red spot' (e).

measures ANOVA). The vitreous glycine level decreased by $50.2 \%$ in the control eye, while it increased by $29.4 \%$ in the CRAO eye; however, the difference did not reach statistical significance ( $P=0.11$, repeated measures ANOVA). The vitreous GABA levels increased by $132 \%$ in the control eye, while it decreased by $37 \%$ in the CRAO eye. This difference also did not reach statistical significance ( $P=0.18$, repeated measures ANOVA), partly because of the very low concentrations of baseline GABA $(=2.0 \mathrm{nmol} / \mathrm{ml})$. A power analysis indicated 4.6and 3.8-fold changes in the vitreous glutamate and glycine concentrations were needed respectively to reach statistical significance $(P<0.05$, power of 0.8 , Table 1$)$. For other amino acids except for GABA and threonine, the power was sufficient to detect 1.3-1.6-fold changes in the study eye (Table 1). There was no significant difference in the retinal amino-acid levels (following CRAO) between the two eyes $(P=0.07-0.93$, paired $t$-test, Table 1$)$. In particular, the retinal glutamate concentrations were 68.9 and 35.9 (nmol/mg protein) in the control and study eye respectively ( $P=0.16$, paired $t$-test). The retinal glycine concentrations were 25.3 and 43.7 ( $\mathrm{nmol} / \mathrm{mg}$ protein) in
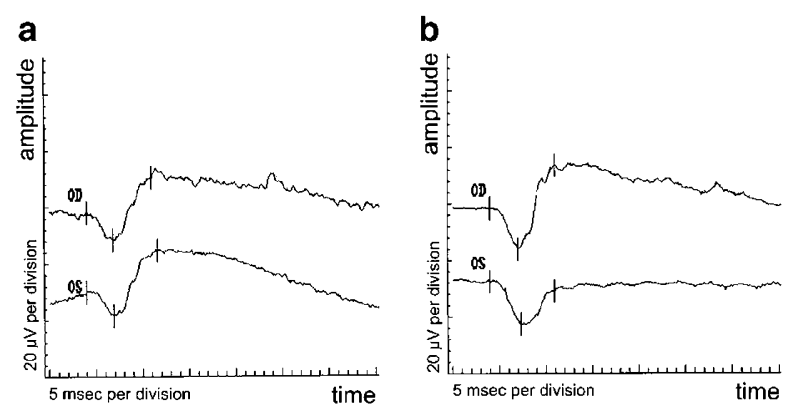

Figure 2 Full-field scotopic electroretinogram before (a) and during CRAO in the left eye (b). (a) Normal full-field scotopic electroretinograms in both eyes. A-wave: $-50.2 \mu \mathrm{V}$ at $14.0 \mathrm{~ms}$ OD, $-23.9 \mu \mathrm{V}$ at $14.5 \mathrm{~ms}$ OS. B-wave: $60.0 \mu \mathrm{V}$ at $34.5 \mathrm{~ms}$ OD, $92.2 \mu \mathrm{V}$ at $38.0 \mathrm{~ms}$ OS. (b) Absence of the b-wave during CRAO OS. A-wave: $-71.5 \mu \mathrm{V}$ at $15.0 \mathrm{~ms}$ OD, $-79.5 \mu \mathrm{V}$ at $16.5 \mathrm{~ms}$ OS. Bwave: $76.6 \mu \mathrm{V}$ at $34.5 \mathrm{~ms} \mathrm{OD},-20.5 \mu \mathrm{V}$ at $34.5 \mathrm{~ms}$ OS. The first marking represents the onset of light stimulus flash. The second and third markings represent the times of a- and b-wave amplitude measurements respectively (\#M20083). a

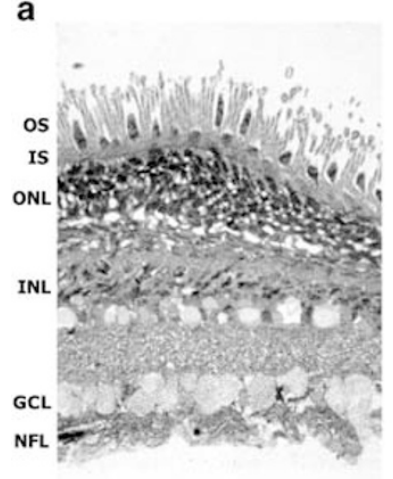

b

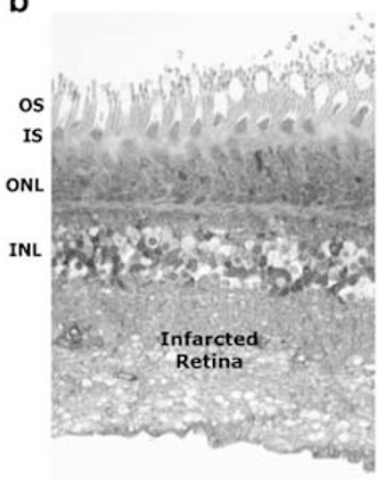

Figure 3 Thin $5 \mu \mathrm{m}$ retinal sections stained with methylene blue. The control retina (a) shows relatively intact retinal cytoarchitecture. Preparation artifact occurred during tissue processing in the inner and outer nuclear layers. The ganglion cell nuclei are stained lightly. In contrast, the study (CRAO) retina (b) depicts characteristic changes of acute inner retinal ischemia. The nerve fibre layer is thickened. Numerous ganglion cell axons are dilated and show lucent axoplasm. Within the inner nuclear layer, cell bodies are enlarged with lucent cytoplasm. The outer retina, including the outer nuclear layer, and inner and outer segments, appear normal with no evidence of ischaemia, necrosis, or atrophy (\#M20068). OS: photoreceptor outer segment; IS: photoreceptor inner segment; ONL: outer nuclear layer; INL: inner nuclear layer; GCL: ganglion cell layer; NFL: nerve fibre layer.

the control and study eye respectively $(P=0.64$, paired $t$ test). A Bonferroni adjustment for multiple comparisons did not alter the statistical results for Table 1 .

We decided to obtain no more than two vitreous samples (one at baseline and the other at the end of the experiment, see Materials and methods) because of the 
inherent risks associated with multiple vitrectomies (such as vitreous haemorrhage and retinal detachment) and the potential for confounding amino-acid results. In one animal, however, we obtained multiple vitreous samples to determine vitreous glutamate concentrations both during and after CRAO. The vitreous glutamate concentrations were slightly lower in the study eye than in the control eye both during and after CRAO (Table 2).

\section{Discussion}

A primate model of CRAO, which closely mimics the human condition, was used to determine whether glutamate excess and excitotoxicity occur in acute retinal ischaemia. This study was undertaken as a part of an effort to develop a rational, neuroprotective strategy for the treatment of acute CRAO and retinal ischaemia.

The ophthalmoscopic fundus appearance coupled with defective retinal arterial filling in the fluorescein angiograms, reduction of the b-wave in the ERG, and the retinal histopathology confirmed the intended occlusion of the CRA and resultant inner retinal ischaemia.

Previous experimental studies in Rhesus monkeys have shown that CRAO for less than 100 min produced little or no apparent retinal damage. However, CRAO of 105240 min produced a variable degree of damage, while almost total irreversible damage was observed with CRAO for longer than 240 min. ${ }^{1,2}$ Based on this, we occluded the central retinal artery for $190 \mathrm{~min}$, well beyond the average retinal tolerance time of $105 \mathrm{~min}$, but still within the time period in which potential therapeutic intervention could be applied. We reasoned that a prolonged retinal ischaemic insult of $190 \mathrm{~min}$ would likely lead to significant glutamate release and associated neural toxicity. ${ }^{7}$ However, we were unable to detect any significant increase in the vitreous or retinal glutamate concentrations following $190 \mathrm{~min}$ of CRAO and $350 \mathrm{~min}$ of reperfusion compared to the control eye (Table 1).

In previous experimental studies, acute retinal ischaemia was produced in lower animals (rat, cat and rabbit) by elevation of the intraocular pressure (IOP) to above the systolic arterial blood pressure level, which produced global ocular ischaemia rather than just inner retinal ischaemia. In those experimental studies of global ocular ischaemia, conflicting results regarding changes in the vitreous glutamate concentration have been reported. (1) In the rat, a global ocular ischaemia was produced by $60 \mathrm{~min}$ of IOP elevation followed by $30 \mathrm{~min}$ of reperfusion. ${ }^{14}$ After $30 \mathrm{~min}$ of reperfusion the vitreous glutamate concentration was 2.2-fold higher in the study eye compared to the control. (2) In the cat, a global ocular ischaemia was produced by $60 \mathrm{~min}$ of IOP elevation followed by $100 \mathrm{~min}$ of re-perfusion. ${ }^{15}$ In this study, the vitreous amino-acid concentration was continuously monitored by a microdialysis probe positioned in the vitreous cavity. At $60-70 \mathrm{~min}$ of reperfusion, the vitreous glutamate concentration peaked at 6.7 times the baseline level. At $90 \mathrm{~min}$ of reperfusion, the vitreous glutamate concentration decreased somewhat but was still greater than five times the baseline level. The vitreous aminoacid concentration was not measured beyond $90 \mathrm{~min}$ of reperfusion. Other studies, however, show only transient or no elevation of vitreous glutamate concentration following global ocular ischaemia. (3) In the rabbit, a global ocular ischaemia was produced by $45 \mathrm{~min}$ of IOP elevation followed by $1 \mathrm{~h}$ of reperfusion. ${ }^{16}$ The glutamate concentration was continuously monitored by a microdialysis probe in the vitreous cavity. There was a transient 5-10-fold increase in the vitreous glutamate concentration during the first $30 \mathrm{~min}$ of the reperfusion. However, after $1 \mathrm{~h}$ of reperfusion the glutamate concentration fell below the baseline level (a 54\% decrease). This study also showed lower glutamate concentration in the ischaemic retinal tissue compared to the control, similar to the current results. (4) Another study produced global ocular ischaemia in the rabbit by $45 \mathrm{~min}$ of either IOP elevation or optic nerve ligation, followed by $2 \mathrm{~h}$ of reperfusion. ${ }^{17}$ The vitreous glutamate concentration was continuously monitored by microdialysis. This study, however, failed to detect even a transient elevation of vitreous glutamate concentration at any point during or following ischaemia. Their results are consistent with the current study.

There are potentially several reasons why the current results are different from those of previous studies. (1) It is possible that the glutamate concentration depends on the exact location of the vitreous biopsy. Since excess glutamate is believed to be released from the ischaemic cells in the retina, it is reasonable to expect a relatively higher vitreous glutamate concentration closer to the retina. We obtained posterior vitreous samples as close to the retinal surface (over the macula) as surgical possible without damaging the internal limiting membrane or retina. There was no systematic difference in the location of the vitreous biopsy between the control and study eye. Finally, the glutamate concentration in the ischaemic retina was actually lower than that of the control retina (Table 1). (2) It is possible that vitreous glutamate concentration was affected by the vitrectomy itself or by the Balanced Salt Solution used subsequently to reform the eye. In order to avoid vitreous dilution, we did not use any irrigating solution during the vitrectomy. Instead, after the vitreous biopsy, equal amount of Balanced Salt Solution (containing no amino acid) was infused into the anterior chamber in both eyes through a separate paracentesis wound in order to reform the globe. This may have led to dilution of vitreous aminoacid concentrations in subsequent vitreous biopsies. 
Table 1 Vitreous and retinal amino acid concentrations

\begin{tabular}{|c|c|c|c|c|c|c|c|}
\hline \multirow[t]{2}{*}{ Amino acid } & \multicolumn{2}{|c|}{ Baseline vitreous concentration } & \multicolumn{2}{|c|}{ Change in vitreous concentration } & \multirow{2}{*}{$\begin{array}{l}\text { Power analysis for change } \\
\text { in vitreous concentration } \\
\text { to reach significance } \\
(P<0.05, \text { power } 0.8) \\
\text { Ratio (post/pre-CRAO) } \\
\text { in study eye* }\end{array}$} & \multicolumn{2}{|c|}{ Retinal concentration } \\
\hline & $\begin{array}{l}\text { Control eye } \\
(\mathrm{nmol} / \mathrm{ml})\end{array}$ & $\begin{array}{l}\text { Study eye (nmol/ } \\
\mathrm{ml})\end{array}$ & Control eye (\%) & Study eye (\%) & & $\begin{array}{c}\text { Control eye } \\
\text { (nmol/mg protein) }\end{array}$ & $\begin{array}{l}\text { Study eye (nmol/ } \\
\text { mg protein) }\end{array}$ \\
\hline ASN & $23.0 \pm 8.4$ & $21.1 \pm 5.4$ & $-30.7 \pm 16.0$ & $-40.1 \pm 7.2$ & 1.4 & $3.9 \pm 1.9$ & $2.7 \pm 1.8$ \\
\hline GABA & $1.6 \pm 1.3$ & $2.0 \pm 1.0$ & $+132.0 \pm 232.1$ & $-37.0 \pm 35.6$ & 8.3 & $15.4 \pm 2.4$ & $5.3 \pm 2.8$ \\
\hline GLN & $992.3 \pm 848.4$ & $954.7 \pm 910.9$ & $-14.7 \pm 24.0$ & $-0.6 \pm 18.0$ & 1.6 & $30.5 \pm 12.6$ & $36.0 \pm 28.5$ \\
\hline GLU & $10.3 \pm 6.3$ & $6.2 \pm 3.8$ & $-34.5 \pm 46.7$ & $-20.5 \pm 7.9$ & 4.6 & $68.9 \pm 23.1$ & $35.9 \pm 24.5$ \\
\hline GLY & $25.0 \pm 15.0$ & $12.6 \pm 7.5$ & $-50.2 \pm 13.9$ & $+29.4 \pm 66.5$ & 3.8 & $25.3 \pm 10.2$ & $43.7 \pm 40.6$ \\
\hline HIS & $18.4 \pm 5.1$ & $18.3 \pm 4.6$ & $-26.9 \pm 12.6$ & $-35.3 \pm 5.0$ & 1.5 & $2.3 \pm 0.6$ & $1.7 \pm 1.2$ \\
\hline ILEU & $30.7 \pm 26.6$ & $26.5 \pm 18.1$ & $+12.8 \pm 26.5$ & $+8.7 \pm 20.3$ & 1.3 & $3.1 \pm 0.7$ & $2.5 \pm 1.7$ \\
\hline LEU & $82.2 \pm 50.2$ & $74.2 \pm 33.5$ & $+3.0 \pm 25.9$ & $-2.9 \pm 21.5$ & 1.4 & $8.3 \pm 2.6$ & $6.3 \pm 4.2$ \\
\hline METH & $18.3 \pm 9.4$ & $18.4 \pm 8.3$ & $-66.8 \pm 7.3$ & $-66.8 \pm 8.4$ & 1.6 & $2.9 \pm 0.8$ & $2.2 \pm 1.8$ \\
\hline PHE & $24.1 \pm 17.7$ & $21.6 \pm 11.6$ & $-24.6 \pm 17.1$ & $-31.5 \pm 9.5$ & 1.5 & $3.6 \pm 0.8$ & $2.3 \pm 1.5$ \\
\hline SER & $100.3 \pm 80.1$ & $70.8 \pm 36.3$ & $-35.2 \pm 6.4$ & $-19.7 \pm 11.1$ & 1.3 & $11.3 \pm 2.3$ & $8.8 \pm 5.9$ \\
\hline THR & $26.2 \pm 35.8$ & $24.4 \pm 22.3$ & $-10.7 \pm 13.1$ & $-19.7 \pm 43.7$ & 4.3 & $27.2 \pm 19.0$ & $18.3 \pm 15.3$ \\
\hline \multirow[t]{2}{*}{ TYR } & $26.8 \pm 24.0$ & $23.0 \pm 16.1$ & $-42.1 \pm 4.2$ & $-44.9 \pm 7.3$ & 1.4 & $2.9 \pm 1.3$ & $2.4 \pm 1.8$ \\
\hline & \multicolumn{2}{|c|}{ Paired $t$-test: $P$-values $0.22->0.99$} & \multicolumn{2}{|c|}{$\begin{array}{c}\text { Repeated measures ANOVA: } P \text {-values } \\
0.09-0.82\end{array}$} & $\begin{array}{l}\text { *Assumes no change in } \\
\text { control eye }\end{array}$ & \multicolumn{2}{|c|}{ Paired t-test: $P$-values $0.07-0.93$} \\
\hline
\end{tabular}

In each of the comparisons, there was no significant difference in the amino-acid levels between the control and study eye.

The numbers are represented as mean $\pm 1 \mathrm{SD}$. 
Table 2 Vitreous glutamate concentrations before, during and after CRAO

\begin{tabular}{lcc}
\hline Time of vitreous biopsy & $\begin{array}{c}\text { Control eye } \\
(\mathrm{nmol} / \mathrm{ml})\end{array}$ & $\begin{array}{c}\text { Study eye } \\
(\mathrm{nmol} / \mathrm{ml})\end{array}$ \\
\hline Pre-CRAO & 7.2 & 7.2 \\
0.7 h of CRAO & 4.8 & 4.2 \\
3.1 h of CRAO & 5.9 & 4.3 \\
0.8 h of reperfusion & 5.3 & 5.1 \\
2.6 h reperfusion & 6.9 & 6.6 \\
5.0 h of reperfusion & 7.4 & 5.5 \\
\hline
\end{tabular}

The vitreous glutamate concentrations were lower in the study eye than in the control eye during and after CRAO (\#M20084).

However, if this were true, we would have expected all, not just some, amino acids to be affected. While most amino-acid concentrations in the control eye indeed decreased on the second biopsy, leucine, isoleucine, and GABA did not and actually showed mean increase (Table 1). Furthermore, we used the fellow eye as a control to compensate for any potential confounding effect of the vitrectomy and vitreous dilution. (3) It is possible that the vitreous glutamate concentration increase is only transient during the initial phase of the blood reperfusion following ocular ischaemia, as suggested by a previous study. ${ }^{16}$ In the current study, we obtained the vitreous sample after $5-6 \mathrm{~h}$ of reperfusion following CRAO and thus, may have 'missed' the transient rise in the vitreous glutamate concentration. We addressed this issue by obtaining multiple vitreous samples both during and following CRAO in one animal. We did not find elevated concentration of vitreous glutamate either during or after CRAO compared to the baseline level or to the control eye (Table 2). Our results are consistent with those of Muller et al. ${ }^{17}$ who found no elevation of vitreous glutamate during ocular ischaemia and up to $2 \mathrm{~h}$ of reperfusion in the rabbit, but inconsistent with those of Louzada-Junior et al. ${ }^{16}$ We acknowledge our results are from a single animal, and it may have been preferable to be able to continuously monitor vitreous amino-acid levels in all four animals. (4) The current experimental model of retinal ischaemia is more selective than any of the previous animal models. Specifically, the current primate model produced only the inner retinal ischaemia by transiently occluding the central retinal artery (see Figures 1-3), while the previous studies. ${ }^{14,15}$ produced more global ocular ischaemia by occluding the entire ocular blood supply. Thus, it is possible that vitreous glutamate elevation is only associated with much more global ocular ischaemia that affects not only the retinal blood supply but also the choroidal and even the anterior segment blood circulation. For example, one might speculate a more limited retinal ischaemia such as CRAO may allow enough blood supply to the Müller cell to scavenge excess extracellular glutamate through a reuptake mechanism, ${ }^{18,19}$ while more complete ocular ischaemia may not. This hypothesis is consistent with a primate study that showed the vitreous glutamate concentration at 12 weeks after partial optic nerve transection (which would affect only the ganglion cell and nerve fibre layers) was not elevated compared to the control eye. ${ }^{20}$ Another primate study also failed to find elevated vitreous glutamate levels in an experimental model of chronic glaucoma in which there is intraocular pressure-dependent retinal ganglion cell and optic nerve damage. $^{21}$

Both the vitreous and retinal glycine showed small increase in the study eye compared to the baseline and control respectively, but the differences were not statistically significant ( $P$-values of 0.11 and 0.64 respectively, Table 1). In a rabbit global ocular ischaemia model, Müller et al ${ }^{17}$ reported vitreous glycine concentration increase by over $50 \%$ after IOP elevation, and over $150 \%$ after optic nerve ligature. In a rat ocular ischaemia model, Lagreze et al ${ }^{14}$ reported $428 \%$ greater vitreous glycine concentration in the study eye compared to the control eye. On the other hand, in a similar rabbit ocular ischaemia model, Louzada et $a l^{16}$ reported no significant change in the vitreous concentration of glycine. Glycine is an inhibitory neurotransmitter in the retina $^{6,22}$ and can also bind to coactivate the N-methyl-Daspartate (NMDA) receptor. ${ }^{7}$ Presently, the role of glycine in ocular or retinal ischaemia remains unclear. Both the vitreous and retinal GABA concentrations showed decrease in the study eye compared to the baseline and control respectively, but the differences were not statistically significant $(P$ - values of 0.18 and 0.11 respectively, Table 1 ). These results are consistent with a previous rat ocular ischaemia study that found no significant difference in the vitreous GABA concentrations between the control and study eye. $^{14}$

There are several limitations in the current study. (1) The number of animals studied was small and consequently limited the power of comparison, especially for the vitreous glutamate, glycine, and GABA (Table 1). In particular, anything less than 4.6-fold relative increase in the vitreous glutamate concentration in the study eye would have been considered statistically insignificant in our experimental design. We observed $14 \%$ relative increase in the vitreous glutamate concentration in the study eye (Table 1). While statistically insignificant, it is possible the small relative increase may be physiologically significant in mediating the inner retinal damage. Likewise, the lower retinal glutamate concentration in the study eye compared to the control eye (Table 1) may not necessarily rule out the role of glutamate, because the entire retinal glutamate 
level (as analysed in the study) may not be indicative of the more localized glutamate level of the ischemic inner retina. (2) The vitreous amino acid levels in the control eye changed from the initial to subsequent biopsy. Four amino acids (GABA, Gly, Meth, Tyr) showed greater than $40 \%$ change, while three amino acids (Asn, Glu, Ser) showed between 30 and $40 \%$ change from the baseline (Table 1). The rest (Gln, His, Ileu, Phe, Thr) showed between 10 and 30\% change, except for Leu which showed only 3\% change from the baseline. Based on our reliability of amino-acid measurements (see Materials and methods above), up to $10 \%$ of the variation could have been due to the repeated measurement variability alone. Additional changes may be attributed to vitrectomy procedure itself or to transient hypotony resulting from removing a small sample of vitreous humour. In order to compensate for any potential confounding effect of the vitrectomy itself, however, we compared the changes in the amino acid level between the study and control eye to determine a relative change attributable to the CRAO. Finally, based on previous studies we anticipated (but did not observe) changes of vitreous glutamate concentration to be 2-6-fold higher during ischaemia, ${ }^{14,15}$ which is well beyond the change seen in the control eye $(-35 \%$, Table 1$)$. (3) It is possible that a more prolonged ischaemia (eg beyond $3 \mathrm{~h}$ of CRAO) may have produced a greater damage and perhaps, rise in vitreous glutamate. Alternatively, waiting a longer period (greater than $6 \mathrm{~h}$ ) following the reperfusion of the retina may have produced a greater reperfusion injury and rise in vitreous glutamate. We established our experimental protocol after reviewing previous studies and according to our clinical experience in managing human patients with acute CRAO. None of the previous reports studied beyond $2 \mathrm{~h}$ of reperfusion..$^{15,14,16}$ In addition, it is a rare patient who would recover vision after more prolonged period of CRAO or delayed presentation after the event. ${ }^{4,5}$ Therefore, we felt the protocol chosen for the study was reasonable, especially given the small number of subjects involved.

In conclusion, while previous animal studies suggest that more global ocular ischaemia may produce significantly elevated vitreous glutamate levels leading to neural toxicity, ${ }^{14-16}$ the current study could not detect significant increase in the vitreous and retinal concentrations of glutamate following acute CRAO in a primate model, that closely models the human condition. Therefore, we conclude the inner retinal ischaemia associated with CRAO may be insufficient to generate detectable glutamate excess that can lead to excitotoxicity. Further studies are warranted to elucidate the role of glutamate excitotoxicity in human clinical conditions of retinal ischaemia.

\section{Acknowledgements}

We acknowledge the excellent technical support provided by Ms Donna McAllister and Trish Duffel. This work was supported in part by University of Iowa College of Medicine Research Award, the Shaffer International Research Fellowship from Glaucoma Research Foundation, and unrestricted grant from Research to Prevent Blindness, New York, New York. $\mathrm{SSH}$ is a Research to Prevent Blindness Senior Scientific Investigator.

\section{References}

1 Hayreh SS, Jonas JB. Optic disk and retinal nerve fiber layer damage after transient central retinal artery occlusion: an experimental study in Rhesus monkeys. Am J Ophthalmol 2000; 129: 786-795.

2 Hayreh SS, Kolder HE, Weingeist TA. Central retinal artery occlusion and retinal tolerance time. Ophthalmology 1980; 87: 75-78.

3 Ffytche TJ. A rationalization of treatment of central retinal artery occlusion. Trans Ophthalmol Soc UK 1974; 94: 468-479.

4 Duker JS, Brown GC. Recovery following acute obstruction of the retinal and choroidal circulations. A case history. Retina 1988; 8: 257-260.

5 Mangat HS. Retinal artery occlusion. Surv Ophthalmol 1995; 40: $145-156$.

6 Pycock CJ. Retinal neurotransmission. Surv Ophthalmol 1985; 29: 355-365.

7 Choi DW. Glutamate neurotoxicity and diseases of the nervous system. Neuron 1988; 1: 623-634.

8 Bresnick GH. Excitotoxins: a possible new mechanism for the pathogenesis of ischemic retinal damage. Arch Ophthalmol 1989; 107: 339-341.

9 Mosinger JL, Price MT, Bai HY, Xiao H, Wozniak DF, Olney JW. Blockade of both NMDA and non-NMDA receptors is required for optimal protection against ischemic neuronal degeneration in the in vivo adult mammalian retina. Exp Neurol 1991; 113: 10-17.

10 Gupta LY, Marmor MF. Mannitol, dextromethorphan, and catalase minimize ischemic damage to retinal pigment epithelium and retina. Arch Ophthalmol 1993; 111: 384-388.

11 Hayreh SS, Weingeist TA. Experimental occlusion of the central artery of the retina. I. Ophthalmoscopic and fluorescein fundus angiographic studies. $\mathrm{Br} J$ Ophthalmol 1980; 64: 896-912.

12 Russell SR, Hageman GS. Optic disc, foveal, and extrafoveal damage due to surgical separation of the vitreous. Arch Ophthalmol 2001; 119: 1653-1658.

13 Miller MW, Waziri R, Baruah S, Gilliam DM. Long-term consequences of prenatal cocaine exposure on biogenic amines in the brains of mice: the role of sex. Brain Res Dev Brain Res 1995; 87: 22-28.

14 Lagreze WA, Knorle R, Bach M, Feuerstein TJ. Memantine is neuroprotective in a rat model of pressure-induced retinal ischemia. Invest Ophthalmol Vis Sci 1998; 39: 1063-1066.

15 Adachi K, Kashii S, Masai H, Ueda M, Morizane C, Kaneda $\mathrm{K}$ et al. Mechanism of the pathogenesis of glutamate neurotoxicity in retinal ischemia. Graefes Arch Clin Exp Ophthalmol 1998; 236: 766-774. 
16 Louzada-Junior P, Dias JJ, Santos WF, Lachat JJ, Bradford HF, Coutinho-Netto J. Glutamate release in experimental ischaemia of the retina: an approach using microdialysis. J Neurochem 1992; 59: 358-363.

17 Muller A, Villain M, Bonne C. The release of amino acids from ischemic retina. Exp Eye Res 1997; 64: 291-293.

18 Napper GA, Pianta MJ, Kalloniatis M. Reduced glutamate uptake by retinal glial cells under ischemic/hypoxic conditions. Vis Neurosci 1999; 16: 149-158.

19 Kawasaki A, Otori Y, Barnstable CJ. Muller cell protection of rat retinal ganglion cells from glutamate and nitric oxide neurotoxicity. Invest Ophthalmol Vis Sci 2000; 41: 3444-3450.

20 Levkovitch-Verbin H, Quigley HA, Kerrigan-Baumrind LA, D'Anna SA, Kerrigan D, Pease ME. Optic nerve transection in monkeys may result in secondary degeneration of retinal ganglion cells. Invest Ophthalmol Vis Sci 2001; 42: 975-982.

21 Carter-Dawson L, Crawford ML, Harwerth RS, Smith III EL, Feldman R, Shen FF et al. Vitreal glutamate concentration in monkeys with experimental glaucoma. Invest Ophthalmol Vis Sci 2002; 43: 2633-2637.

22 Kalloniatis M. Amino acids in neurotransmission and disease. J Am Optom Assoc 1995; 66: 750-757. 